\title{
Fundamental Architecture and Analysis of an Antimatter Ultra-Intense Laser Derived Pulsed Space Propulsion System
}

\author{
Robert Le Moyne1, Timothy Mastroianni2 ${ }^{2}$ \\ ${ }^{1}$ Independent Author (Senior Member AIAA \& Senior Member IEEE), Running Springs, California, USA \\ ${ }^{2}$ Independent Author, Pittsburgh, Pennsylvania, USA \\ Email: rlemoyne07@gmail.com
}

Received December 2013

\section{Abstract}

Antimatter has been generated in large quantities by the Lawrence Livermore National Laboratory Titan laser. The Titan laser is an ultra-intense laser system on the order of approximately $10^{20} \mathrm{~W} / \mathrm{cm}^{2}$ with pulse durations of roughly $1 \mathrm{ps}$. With the Titan laser incident on a high atomic number target, such as gold, antimatter on the scale of $2 \times 10^{10}$ positrons are generated. Roughly $\mathbf{9 0 \%}$ of the generated positrons are ejected anisotropic and aft to the respective target. The mechanisms for the laser-derived positron antimatter generation involve electron interaction with the nuclei based on bremsstrahlung photons that yield electron-positron pairs as a consequence of the Bethe-Heitler process, which predominates the Trident process. Given the constraints of the current and near future technology space, a pulsed space propulsion configuration is advocated for antimatter derived space propulsion, similar in concept to pulsed radioisotope propulsion. Antimatter is generated through an ultra-intense laser on the scale of a Titan laser incident on a gold target and annihilated in a closed chamber, representative of a combustion chamber. Upon reaching a temperature threshold, the closed chamber opens, producing a pulse of thrust. The implication of the pulsed space propulsion antimatter architecture is that the energy source for the antimatter propulsion system can be decoupled from the actual spacecraft. In contrast to conventional chemical propulsion systems, which require storage of its respective propulsive chemical potential energy, the proposed antimatter propulsion architecture may have the energy source at a disparate location from the spacecraft. The ultra-intense laser could convey its laser energy over a distance to the actual spacecraft equipped with the positron antimatter pulsed space propulsion system. Hydrogen is considered as the propulsive fluid, in light of its low molecular weight. Fundamental analysis is applied to preliminarily define the performance of the positron antimatter derived pulsed space propulsion system. The fundamental performance analysis of the antimatter pulsed space propulsion system successfully reveals the architecture is viable for further evaluation.

\section{Keywords}

Ultra-Intense Laser, Antimatter, Positron, Antimatter Propulsion, Antimatter Generation, Space Propulsion 


\section{Introduction}

The progressive evolution of fundamental science enables a novel architecture for antimatter propulsion. Rather than incorporating traditional concepts which require evolution of other technologies, such as magnetic confinement or geometric constraints that inherently would require assembling in low earth orbit conditions; the proposed architecture for antimatter propulsion may be readily tested and evaluated given the current technology space. The antimatter propulsion concept incorporates a pulsed space propulsion format, which is far more feasible than that of a steady state antimatter propulsion system. The positron antimatter pulsed space propulsion architecture incorporates the current technology readiness involving the application of ultra-intense lasers. The positron antimatter pulsed space propulsion architecture is envisioned for small spacecraft primary maneuvering and attitude control.

Roughly a quarter of a century ago a conceptual design for an antimatter rocket based combined cycle was promoted. However, viable antimatter production and storage technologies were and are currently unavailable for such a conceptual design [1]. Steady state architecture incorporating antimatter generation from ultra-intense lasers for ramjet applications has been successfully proposed by LeMoyne. The storage requirements are also alleviated as antimatter, in the form of positrons, are only generated by the incidence of an ultra-intense laser [2].

The capacity to generate significant quantities of positron antimatter as a consequence of ultra-intense lasers has steadily progressed over the course of approximately four decades [3-5]. Current ultra-intense laser applications have demonstrated the ability to generate on demand and in-situ antimatter with laser incidence on a high atomic number target, such as gold [4] [5]. However, the pulse rate requirement for steady state applications warrants future evolution of ultra-intense laser technology.

The pulsed space propulsion condition alleviates the need for future ultra-intense laser pulse rate technology evolution. Pulsed propulsion has been proposed for other configurations of non-chemical propulsion. For example radioactive isotopes with protracted half-lives can accumulate thermal energy as a consequence of radioactive decay. Upon the acquisition of a stored thermal threshold, the pulsed space propulsion system releases accumulated propulsive force [6] [7].

A fundamental performance analysis of positron antimatter laser derived pulsed space propulsion is established. The fundamental performance analysis incorporates isentropic and energy balance relations. The positron antimatter laser derived pulsed space propulsion application involves an ultra-intense laser incident on a gold target for the generation of antimatter. The generated antimatter annihilates to accumulate thermal energy for the propulsive thrust. Prior to the performance analysis and propulsion system description, the foundation for positron antimatter generation from the incidence of ultra-intense lasers is presented. Contrast of the proposed architecture to previous antimatter propulsion systems is established.

Appreciable on-spacecraft production and storage of antimatter may affect the consequence of escalated system complexity. By contrast the proposed architecture for pulsed positron antimatter space propulsion conveys laser energy to a target on the propulsion system, thereby decoupling the laser energy source from the spacecraft. The proposed architecture also generates antimatter on demand and in-situ to the pulsed space propulsion system, negating the complexity of antimatter storage requirements.

\section{Previous Antimatter Propulsion Architectures}

Antimatter derived propulsion has been a topic of interest for aerospace propulsion applications, in light of its energy density. Antimatter-matter annihilation constitutes the greatest liberation of energy available [8]. One gram of antimatter is comparable to the amount of energy stored in 23 Space Shuttle External Tanks [9] [10]. An important consideration prior to addressing previous theoretical antimatter propulsion architectures is the disparity between the positron and the antiproton.

The positron and the antiproton constitute two different classes of antimatter. The positron annihilates with an accompanying electron, yielding two gamma ray photons on the scale of $1.02 \mathrm{MeV}$ [11-13]. The positron was discovered in 1936 [14]. The antiproton annihilates with a proton yielding pions. Neutral pions quickly decay into approximately $200 \mathrm{MeV}$ by gamma radiation [15]. A portion of the resultant pions is charged [12] [15]-[18]. The decay pathway for the charged pions results in muons and neutrinos [16] [18]. However, pions decay after a displacement about 21 meters [15] [19] [20].

A major difference between positrons and antiprotons is in consideration of their generation and storage. Po- 
sitrons can be produced by the incidence of an ultra-intense laser on a high atomic number target, such as gold. Positrons can be generated on demand, with incidence of the ultra-intense laser on a gold target [2]-[5].

By contrast, particle accelerators generally produce antiprotons, such as through the facilities at CERN and Fermi National Accelerator Laboratory. However, production is insufficient for envisioned propulsion applications. Also storage technology, although nascent, is insufficient for an antiproton antimatter propulsion application [12].

Multiple conceptual architectures have been developed for the eventual goal of reaching antimatter propulsion predominantly regarding antiprotons. During the 1980's Dr. Forward of Hughes Research Laboratory proposed a space propulsion system incorporating antimatter annihilation. The propulsion system incorporates the production and storage of antimatter, local to the spacecraft. Proposed methodologies for the storage of antimatter involve the use of electric fields, magnetic fields, and lasers. Intra-solar system applications would involve the collection of solar energy [19]. However, the size and mass magnitude for generating antimatter from solar array energy may diminish feasibility.

Other perspectives in antimatter propulsion were demonstrated in the 1980's. Vulpetti proposed a liquid propellant thermal antimatter engine (LIPTHANE). The central theme to the LIPTHANE is the use of a heat converter transmitter system that features a high atomic number, which incorporates gaseous xenon. According to the design parameters, LIPTHANE requires an antiproton flux of $10^{17}$ per second. A major drawback cited is that even laboratory settings fluxes are several orders of magnitude less than the LIPTHANE antiproton flux requirements [21]. Also the LIPTHANE propulsion system lacks viable antiproton storage to support the necessary flux.

Froning during 1988 proposed the use of antiproton antimatter for an air-breathing propulsion configuration. A goal of the configuration was to reduce propellant mass requirements. The antiproton production strategy was based on particle accelerator technology. An extrapolation of particle acceleration technology anticipated that by roughly 2013, a sufficient amount of antiproton production threshold would be attained [1]. However, sufficient antiproton production from particle accelerator technology has yet to be realized. Another issue with storing large quantities of antimatter for a launch vehicle is the safety risk [1]. Also, human occupation of an antimatter based launch vehicle applications involve the inherent risk of gamma radiation exposure [20].

Five identified architectures for antiproton derived antimatter propulsion are: solid core rockets, gaseous core rockets, plasma core rockets, pion rockets, which direct the annihilation products by magnetic fields, and interstellar ramjets. Regarding the solid, gaseous, and plasma core rockets the antiproton annihilation with matter heats the propulsive fluid and is expanded by a nozzle. In the case of the plasma core rocket, a magnetic nozzle is proposed [16]. The pion rocket is conceptualized on the principle that pions could be directed by magnetic fields [16]-[18] [22]. The interstellar ramjet incorporates antiproton annihilation with available interstellar hydrogen [16] [17]. In order to support the quantity of antiprotons necessary for space propulsion missions, substantial advances in the supporting infrastructure for the production of antiproton antimatter are needed [17].

In light of the infrastructure limitations for antiproton production, the concept of using a limited portion of anti-hydrogen antimatter to catalyze inertial fusion has been addressed. The antimatter would be injected into a pellet consisting of deuterium and tritium or lithium deuteride. The pellet would comprise a hemisphere of fissionable uranium or plutonium. The antimatter annihilation would induce temperature levels for localized fusion [9] [23] [24]. Laser compression techniques have been suggested to augment the micro-fusion event. Such architectures have been proposed for missions to Mars [25]. Even though antimatter requirements have been substantially reduced, antimatter catalyzed fusion has not been realized.

Winterberg offers a paradigm shift for the use of antimatter propulsion. Rather than using proton-antiproton annihilation to heat a propulsive fluid for later expansion through a nozzle, the full conversion of the reaction into gamma radiation is considered. The gamma radiation is developed into a coherent gamma ray laser beam. The recoil of the laser beam pulse is transmitted to the spacecraft for propulsion. The novel propulsion concept is considered appropriate for small spacecraft [26].

In consideration of the current perspective for approaching antimatter propulsion there exist substantial challenges: antimatter production, storage, and operation. Enabling technologies, such as facilities with sufficient production rate and antimatter storage systems, not only at the production facility but also appropriate for the environment of the launch vehicle or spacecraft, are imperative for successful implementation [15] [22] [27]. 


\section{Ultra-İntense Laser Derived Antimatter}

The proposed pulsed space propulsion configuration alleviates the technologically unfeasible requirements for storage of antimatter. A recently realized strategy for generating antimatter is presented. The ultra-intense laser is capable of generating antimatter in the form of positrons when incident on a high atomic number target, such as gold. Therefore, the positron generation of antimatter occurs on demand as a consequence of the ultra-intense laser striking the gold target. Simply shutting off the ultra-intense laser ceases antimatter production of positrons; therefore greatly alleviating any antimatter storage requirement. LeMoyne successfully presented a similar concept of antimatter propulsion for ramjet systems during 2012 [2]. In order to further alleviate ultra-intense laser beam pulse requirements, the antimatter space propulsion incorporates a pulsed space propulsion configuration.

During the early 1970's Shearer et al. published a conceptual strategy for the generation of positrons requiring lasers on the scale of approximately $10^{20} \mathrm{~W} / \mathrm{cm}^{2}$ [3]. Three and a half decades later, Lawrence Livermore National Laboratory experimentally demonstrated positron antimatter generation through an ultra-intense short pulse laser incident on a high atomic number target, such as gold. The laser intensity was approximately $10^{20}$ $\mathrm{W} / \mathrm{cm}^{2}$, using pulse duration on the order of $1 \mathrm{ps}$. The ultra-intense laser pulse produced $2 \times 10^{10}$ positrons. Approximately $90 \%$ of the positrons were discharged aft relative to the laser target. The laser target consisted of roughly $1 \mathrm{~mm}$ thick of gold [4] [5]. The ultra-intense laser pulse induced interaction between the electron and nuclei that caused the generation of positron antimatter [4] [5] [28]. The Trident process and Bethe-Heitler process are considered to be the basis for position antimatter generation using a high atomic number nuclei [4] [28].

The Trident process utilizes a single step for generation of an antimatter positron. The electron directly interacts with the nuclei to produce an electron-positron pair.

$$
e^{-}+Z \rightarrow e^{+}+2 e^{-}+Z
$$

$e^{+}:$Positron

$e^{-}:$Electron

$Z$ : High atomic number nuclei(such as gold) [4] [28].

The Bethe-Heitler process comprises two steps. Fast electrons produce high-energy bremsstrahlung photons. Then bremsstrahlung photons interact with the nuclei generating electron positron pairs.

$$
\begin{gathered}
e^{-}+Z \rightarrow \gamma+e^{-}+Z \\
\gamma+Z \rightarrow e^{+}+e^{-}+Z
\end{gathered}
$$

$\gamma$ : Bremsstrahlung photons [4] [28].

The Bethe-Heitler process predominates for antimatter generation, since the Bethe-Heitler process cross section is 100 times greater than the Trident process. Target thickness influences the prevalent electron-positron pair generation process. The Bethe-Heitler process is significant for gold targets approximately $1 \mathrm{~mm}$ thick. The Trident process is the influential for gold targets that are about 3.5 micrometers thick [28].

The Lawrence Livermore National Laboratory Titan laser generated $2 \cdot 10^{10}$ positrons through a $\sim 1$ ps pulse on $\sim 1 \mathrm{~mm}$ gold target. The Titan laser intensity is $\sim 10^{20} \mathrm{~W} / \mathrm{cm}^{2}$ using $\sim 120 \mathrm{~J}$ of energy with a laser beam wavelength of $1054 \mathrm{~nm}$. The Bethe-Heitler process was identified as the most significant positron antimatter generation mechanism. The positron temperature was about $2 \mathrm{MeV}$. Approximately 10 times as many positrons were discharged anisotropically normal to the aft relative the front [4] [5].

The implication of positron antimatter generated by an ultra-intense laser is that the energy source is decoupled from the propulsion system. Traditional spacecraft propulsion systems require the chemical energy source of the propulsion system to be stored and integrated in to the spacecraft structure [29] [30]. The energy source for the antimatter pulse propulsion concept could literally be a terrestrial nuclear reactor with an ultraintense laser apparatus on the scale of a building conveying the ultra-intense laser beam to the targeted spacecraft at a remote distance. The spacecraft with pulsed antimatter propulsion only requires a $\sim 1 \mathrm{~mm}$ thick gold target.

Another notable attribute of the proposed antimatter pulsed space propulsion concept is that the ultra-intense laser generates a series of pulses on the order of 1ps [4] [5]. By contrast published laser propelled light sail configurations require a continuous $1 \mathrm{PW}$ of laser power [17]. Incorporating a pulsed space propulsion architecture further alleviates the pulse requirements for the ultra-intense laser. 


\section{Pulsed Space Propulsion}

The concept of pulsed space propulsion applications involves the progressive storage of thermal energy, by a source such as radioactive decay. The storage of thermal energy occurs for a duration longer than the time span of the propulsive thrust. Pulsed space propulsion designs are considered appropriate for small spacecraft primary maneuvering and attitude control [6] [7]. As opposed to conventional radioactive decay, antimatter annihilation provides the source of stored thermal energy.

\section{Antimatter Derived Pulsed Space Propulsion Systemusing Ultra-İntense Laser}

The envisioned antimatter pulsed space propulsion concept will incorporate a series of antimatter annihilation pulses ultra-intense laser on the scale of a Titan laser incident on a $1 \mathrm{~mm}$ thick gold target to generate positron antimatter. The pulsed space propulsion system is under a closed chamber condition. The propulsive fluid is progressively heated over a duration of time reaching a threshold temperature of $2500 \mathrm{~K}$. Hydrogen is selected as the propulsive fluid because of its low molecular weight. Upon attaining the $2500 \mathrm{~K}$ hydrogen propulsive fluid temperature threshold the chamber opens generating a pulse of thrust.

In case the cross-section for positron antimatter annihilation is insufficient for hydrogen, a contingency configuration is also considered. Tungsten is incorporated into the second design configuration, as its higher atomic number and density may provide better annihilation cross-section properties. The second configuration would incorporate a mass of tungsten internal to the closed chamber. The positron beam would annihilate with the tungsten target (note that positron antimatter not antiprotons is considered), thereby heating the encompassing propulsive hydrogen.

The energy source of the ultra-intense laser system is remote from the spacecraft equipped with the antimatter pulsed space propulsion system. Therefore the spacecraft global architecture does not need to compensate for large tanks of chemical potential energy or solar cells to specifically provide power to propulsion.

\section{Fundamental Analysis and Performance Results}

The objective of conducting fundamental analysis for the proposed antimatter pulsed space propulsion system is to provide preliminary proof of concept. Higher fidelity analyses can be conducted later. The first goal is to establish an energy balance to attain $2500 \mathrm{~K}$ for the hydrogen fluid, and then determine the number of ultra-intense laser pulses required to achieve the temperature threshold. The second goal is to determine steady state propulsion performance parameters.

The Lawrence Livermore National Laboratory ultra-intense Titan laser $\left(10^{20} \mathrm{~W} / \mathrm{cm}^{2}\right)$ incorporates $\sim 1$ ps pulses to generate $2 \times 10^{10}$ positrons by laser incidence on a $\sim 1 \mathrm{~mm}$ thick gold target. Approximately $90 \%$ of the positrons are discharged anisotropic and aft to the laser target. The predominant mechanism is the Bethe-Heitler process. Each $\sim 1$ ps pulse generates $1.8 \times 10^{10}$ positrons to the aft of the laser target with roughly $2 \mathrm{MeV}$ of kinetic energy [4] [5].

The rest mass of a positron and electron pair is $2 \mathrm{mc}^{2}=1.02 \mathrm{MeV}$. The electron rest mass (m) is equivalent to the positron rest mass (m), and c is the speed of light. The kinetic energy of the positron is $2 \mathrm{MeV}$. Collisions are assumed elastic, such that kinetic energy is conserved [11] [31]. The energy of a positron and electron annihilation is $3.02 \mathrm{MeV}$ [4] [5]. For each ultra-intense laser $\sim 1$ ps pulse, $8.71 \times 10^{-6} \mathrm{~kJ}$ of energy is released aft to the laser target.

The temperature prior to antimatter annihilation is assumed to be $300 \mathrm{~K}$. Actual spacecraft reference temperatures could be determined with more sophisticated techniques. Table 1 defines the closed chamber conditions. Table 2 defines the material properties of tungsten and hydrogen [6] [30] [32] [33].

Table 1. Closed chamber conditions.

\begin{tabular}{cc}
\hline Hydrogen temperature (K) & 2500 \\
Hydrogen pressure (atm) & 30 \\
Chamber radius (m) & 0.2 \\
Hydrogen mass (kg) & 0.01 \\
Tungsten plate mass (kg) & 0.1 \\
\hline
\end{tabular}


Table 2. Material properties of tungsten and hydrogen at $2500 \mathrm{~K}$.

\begin{tabular}{cc}
\hline Tungsten specific heat $(\mathrm{kJ} / \mathrm{kg}-\mathrm{K})$ & 0.18 \\
Hydrogen constant pressure specific heat $(\mathrm{kJ} / \mathrm{kg}-\mathrm{K})$ & 17.9 \\
Hydrogen specific heat ratio & 1.2 \\
\hline
\end{tabular}

Assuming an adiabatic surrounding of the chamber, the energy balance for determining the number of ultraintense laser pulses is defined as follows:

$$
Q_{\text {antimatter }}=\left(m_{\text {hydrogen }} c_{p(\text { hydrogen })}+m_{\text {tungsten }} c_{\text {tungsten }}\right)\left(T_{f}-T_{i}\right)
$$

$Q_{\text {antimatter }}:$ Antimatter heat addition

$m_{\text {hydrogen }}:$ Mass of hydrogen

$C_{p(\text { hydrogen })}$ : Specific heat of hydrogen

$m_{\text {tungsten }}:$ Mass of tungsten

$c_{\text {tungsten }}:$ Specific heat of tungsten

$T_{f}$ :Final temperature

$T_{i}$ : Intial temperature

The envisioned antimatter pulsed space propulsion concept consists of solely a closed chamber of hydrogen to be heated by positron antimatter annihilation. Upon reaching a temperature threshold of $2500 \mathrm{~K}$, a pulse of thrust is generated. The required heat addition as a consequence of antimatter annihilation is $386 \mathrm{~kJ}$. A total of $4.4 \times 10^{7}$ pulses from an ultra-intense laser would be required to generate a sufficient quantity of positron antimatter to heat hydrogen to $2500 \mathrm{~K}$.

The contingency configuration incorporates a $0.1 \mathrm{~kg}$ plate of tungsten. The role of the tungsten plate is to augment the functional cross section for positron antimatter annihilation. The addition of the tungsten plate increases the required heat addition by $40 \mathrm{~kJ}$ for a total heat addition by antimatter annihilation to $426 \mathrm{~kJ}$. Regarding the contingency configuration, a total of $4.9 \times 10^{7}$ pulses from an ultra-intense laser would be required to generate a sufficient quantity of positron antimatter to heat hydrogen to $2500 \mathrm{~K}$.

Steady state fundamental propulsion analysis is incorporated to evaluate the propulsion performance parameters of the proposed antimatter pulsed space propulsion system. Both the hydrogen only and contingency with tungsten configuration consist of 10 grams of hydrogen that is pressurized to $30 \mathrm{~atm}$ at $2500 \mathrm{~K}$, so their propulsion performance characteristics are equivalent. Based on a previous non-chemical space propulsion configuration by LeMoyne, the mass flow is set to $1 \mathrm{gram} / \mathrm{second}$ [6]. During the steady state aspect of the propulsive expansion through a nozzle, the following relation, due to synthesis of isentropic relationships, is appropriate for acquiring specific impulse:

$$
I_{s p}=\frac{1}{g}\left\{\frac{2 \gamma R T_{o}}{\gamma-1}\left[1-\left(\frac{p_{e}}{p_{o}}\right)^{(\gamma-1) / \gamma}\right]\right\}^{1 / 2}
$$

$I_{s p}:$ Specific impulse

$g$ : Gravity

$R$ : Specific gas constant

$T_{o}:$ Stagnation temperature of chamber

$p_{o}:$ Stagnation pressure of chamber

$p_{e}:$ Exit pressure

$\gamma$ : Specific heat ra [29].

The above equation for deriving specific impulse assumes that the propulsive flow is expanding to a space environment, and the exhaust pressure has a negligible contribution to the propulsive thrust. For the proposed positron antimatter pulsed space propulsion system with positron generation through ultra-intense laser pulses, the specific impulse is 1140 seconds. The thrust is $11.2 \mathrm{~N}$.

The proposed propulsion configuration offers a robust non-chemical alternative for spacecraft. Incorporating a pulsed space propulsion strategy, as opposed to a continuous mass flow system, alleviates the constraints on the 
ultra-intense laser. Once the pulse of propulsive thrust is expelled, a new mass of hydrogen fluid can be heated from a new series of ultra-intense laser pulses incident on a $\sim 1 \mathrm{~mm}$ gold target that generates positron antimatter. Preliminary investigations can be conducted by directing an ultra-intense laser on a $\sim 1 \mathrm{~mm}$ gold target with the generated positrons annihilating on a working fluid and imparting an energy source on the working fluid. Such an experimental scenario could be evaluated in a laboratory environment with access to an ultra-intense laser.

\section{Feasibility of Antimatter Pulsed Propulsion}

The antimatter pulsed space propulsion constitutes the integration of newly demonstrated laboratory strategies for generating considerable quantities of antimatter through ultra-intense lasers. Intuitively the ultra-intensity laser strategy for antimatter generation could be integrated into a propulsion system. A pulsed space propulsion strategy is incorporated to reduce requirements on the ultra-intense laser.

Other laser derived space propulsion systems have been conceptually developed. The perspective by Winterberg resembles the concept of the laser propelled light sail [26]. The laser propelled light sail uses a laser beam to provide propulsion [8]. Light imparts momentum to the sail, as photons constitute particles of light. The proposed sails functioning as receiver optics have diameters on the scale of $1000 \mathrm{~km}$. Laser power requirements are on the scope of a continuous $1 \mathrm{PW}$ power budget [17]. Such power requirements are at the capacity level of all of civilization [12]. In order to achieve the desired sub-relativistic velocities the propulsive laser beam must be focused on the sail target for years of duration. Although inherently encumbered with substantial geometric and power budget constrains, the laser powered light sail propulsion application has been published as a near term technology [34].

The advanced concept of integrating ultra-intense laser technology as an applied physics breakthrough into a practical aerospace application entails many unknowns and many design challenges. For example the limitations of an ultra-intense laser on a gold target to generate positrons are unknown. The generation of positron antimatter does impart $120 \mathrm{~J}$ of ultra-intense laser energy on a 1mm gold target [4] [5]. Design challenges, such as absorption of resultant antimatter annihilation radiation, optimization of flight characteristics, and analytical consideration of the potentially relativistic effects of the resultant antimatter, should be addressed. A regenerative cooling system to sustain a viable and sustainable temperature for the gold substrate versus the core temperature of the hydrogen propulsive fluid should be applied. Regenerative cooling has been successfully implemented in rocket propulsion systems numerous times [30]. Another strategy to address the thermal constraints would be to apply a magnetic confinement system. The targeting of relativistic particles has been refined to the point of integration into medical technology applications [13] [17] [20] [35]. The future evolutions of ultra-intense lasers are expected, such as improvements in laser energy, pulse rate, and further convergence to the direct process threshold of laser intensity.

\section{Conclusions}

Conventional perspectives in antimatter propulsion have previously advocated production by particle accelerators and a storage system. The conventional approach has been identified as requiring considerable technological investment to reach fruition. Ultra-intense lasers, such as the Lawrence Livermore National Laboratory Titan laser, have been demonstrated for producing substantial amounts of positrons on the order of $2 \times 10^{10}$ are generated by striking a high atomic number target, such as a $\sim 1 \mathrm{~mm}$ gold target. Approximately $90 \%$ of the positrons are ejected anisotropic and aft to the respective target.

The implications of the observed applied physics break through enable a new conceptual strategy for antimatter propulsion using positrons. The positrons could be generated on demand as a function of the activation of the ultra-intense laser, mitigating pending technologies for storage. The ultra-intense laser system could exist remote from the spacecraft, enabling a decoupling of the propulsion system and its propulsive energy source.

The proposed spacecraft positron antimatter propulsion system incorporates a pulse-propulsion format to alleviate ultra-intense laser propulsion requirements. The propulsion applications store energy in hydrogen encased in a closed chamber from positron annihilation. Two configurations are considered: a closed chamber consisting of solely hydrogen that requires $4.4 \times 10^{7}$ pulses from an ultra-intense laser to reach a temperature of $2500 \mathrm{~K}$ for the hydrogen propulsive fluid. A contingency configuration incorporates higher cross-section tungsten with the hydrogen, and the alternative configuration would require $4.9 \times 10^{7}$ pulses from an ultra-intense laser to attain a $2500 \mathrm{~K}$ temperature for hydrogen. Upon reaching the $2500 \mathrm{~K}$ hydrogen temperature threshold, the chamber 
opens generating a pulse of thrust. Fundamental analysis reveals that the positron antimatter propulsion system provides a specific impulse of 1140 seconds with a corresponding thrust of $11.2 \mathrm{~N}$. The positron antimatter pulsed space propulsion architecture is suitable for small spacecraft primary maneuvering and attitude control applications.

As the proposed positron antimatter pulsed space propulsion architecture synthesizes observation of applied physics, many test and evaluation issues remain. For example, are there any limitations to the ultra-intense laser incident on a gold target for a sustained pulse level? Ideally this publication brings more attention to a novel approach for realizing a viable strategy for positron induced antimatter propulsion.

\section{References}

[1] Froning Jr., H.D. (1988) Investigation of Antimatter Air-Breathing Propulsion for Single-Stage-to-Orbit Ships. Acta Astronautica, 17, 853-861. http://dx.doi.org/10.1016/0094-5765(88)90168-3

[2] LeMoyne, R. (2012) Fundamental Architecture and Analysis of Ramjet Propulsion System Utilizing In-Situ Laser Derived Antimatter. Proceedings of $18^{\text {th }}$ AIAA/3AF International Space Planes and Hypersonic Systems and Technologies Conference, AIAA-2012-5863, Tours, France, September.

[3] Shearer, J. W., Garrison, J., Wong, J. and Swain, J. E. (1973) Pair Production by Relativistic Electrons from an Intense Laser Focus. Physical Review A, 8, 1582-1588. http://dx.doi.org/10.1103/PhysRevA.8.1582

[4] Chen, H., Wilks, S.C., Bonlie, J.D., Chen, S.N., Cone, K.V., Elberson, L.N., Gregori, G., Meyerhofer, D.D., Myatt, J., Price, D.F., Schneider, M.B., Shepherd, R., Stafford, D.C., Tommasini, R., Van Maren, R. and Beiersdorfer, P. (2009) Making Relativistic Positrons Using Ultraintense Short Pulse Lasers. Physics of Plasmas, 16. http://dx.doi.org/10.1063/1.3271355

[5] Chen, H., Wilks, S.C., Bonlie, J.D., Liang, E.P., Myatt, J., Price, D.F., Meyerhofer, D.D. and Beiersdorfer, P. (2009) Relativistic Positron Creation Using Ultraintense Short Pulse Lasers. Physical Review Letters, 102. http://dx.doi.org/10.1103/PhysRevLett.102.105001

[6] LeMoyne, R. (2006) Fundamental Analysis of Radioisotope Propulsion. Proceedings of Space 2006, AIAA-2006-7272, San Jose, California, September.

[7] Williams, G.J., Jenkins, R.M. and Rose, M.F. (1994) Preliminary Design of a Space Propulsion System Utilizing Stored Thermal Energy. Journal of Propulsion and Power, 10, 32-39. http://dx.doi.org/10.2514/3.23708

[8] Harris, D., Bille, M. and Reed, L. (2003) Future Space Transportation Technology: Prospects and Priorities. Proceedings of Space 2003, AIAA-2003-6201, Long Beach, California, September.

[9] Schmidt, G.R., Gerrish, H.P. Martin, J.J., Smith, G.A. and Meyer, K.J. (1999) Antimatter Production for Near-Term Propulsion Applications. Proceedings of 35th AIAA, ASME, SAE, and ASEE, Joint Propulsion Conference and Exhibit, AIAA-1999-2691, Los Angeles, California, June.

[10] Derosa, L. and Maccone, C. (2007) Propulsion Tradeoffs for a Mission to Alpha Centauri. ActaAstronautica, 60, 711718. http://dx.doi.org/10.1016/j.actaastro.2006.10.003

[11] Serway, R.A., Moses, C.J. and Moyer, C.A. (1989) Modern Physics. Saunders College, Philadelphia, Pennsylvania.

[12] Frisbee, R.H. (2003) Advanced Propulsion for The XXI Century. Proceedings of AIAA/ICAS International Air andSpace Symposium and Exposition, AIAA-2003-2589, Dayton, Ohio, July.

[13] Haloulakos, V.E. (1996) Antimatter-The Ultimate Energy Source and Its Potential Applications. Proceedings of 32nd AIAA, ASME, SAE, and ASEE, Joint Propulsion Conference and Exhibit, AIAA-1996-2928, Lake Buena Vista, Florida, July.

[14] Fuhs, A. (1990) Future Science and Technology for Military Space. Proceedings of AIAA Space Programs and Technologies Conference, AIAA-1990-3827, Huntsville, Alabama, September.

[15] Frisbee, R.H. (2008) Optimization of Antimatter Rocket Performance. Proceedings of $44^{\text {th }}$ AIAA, ASME, SAE, and ASEE, Joint Propulsion Conference and Exhibit, AIAA-2008-4796, Hartford, Connecticut, July.

[16] Cassenti, B. (1989) Conceptual Designs for Antiproton Space Propulsion Systems. Proceedings of AIAA, ASME, SAE, and ASEE, 25th Joint Propulsion Conference, AIAA-1989-2333, Monterey, California, July.

[17] Frisbee, R.H. and Leifer, S.D. (1998) Evaluation of Propulsion Options for Interstellar Missions. Proceedings of 34th AIAA, ASME, SAE, and ASEE, Joint Propulsion Conference and Exhibit, AIAA-1998-3403, Cleveland, Ohio, July.

[18] Coreano, L. and Cassenti, B.N. (2004) A Comparison of Antimatter Driven Interstellar Propulsion Systems. Proceedings of 40th AIAA, ASME, SAE, and ASEE, Joint Propulsion Conference and Exhibit, AIAA-2004-3705, Fort Lauderdale, Florida, July.

[19] Forward, R.L. (1986) Feasibility of Interstellar Travel: A Review. ActaAstronautica, 14, 243-252. 
http://dx.doi.org/10.1016/0094-5765(86)90126-8

[20] Tarpley, C., Lewis, M.J. and Kothari, A.P. (1990) Safety Issues in SSTO Spacecraft Powered by Antimatter Rocket Engines. Proceedings ofAIAA, ASME, SAE, and ASEE, 26th Joint Propulsion Conference, AIAA-1990-2365, Orlando, Florida, July.

[21] Vulpetti, G. (1987) A Further Analysis About the Liquid-Propellant Thermal Antimatter Engine Design Concept. ActaAstronautica, 15, 551-555. http://dx.doi.org/10.1016/0094-5765(87)90155-X

[22] Frisbee, R.H. (2003) How to Build an Antimatter Rocket for Interstellar Missions-Systems Level Considerations in Designing Advanced Propulsion Technology Vehicles. Proceedings of 39th AIAA, ASME, SAE, and ASEE, Joint Propulsion Conference and Exhibit, AIAA-2003-4676, Huntsville, Alabama, July.

[23] Cassenti, B., Kammash, T. and Galbraith, D. (1994) An Antiproton Catalyzed Inertial Fusion Propulsion System. Proceedings of 30th AIAA, ASME, SAE, and ASEE, Joint Propulsion Conference and Exhibit, AIAA-1994-3354, Indianapolis, Indiana, June.

[24] Cassenti, B.N., Kammash, T. and Galbraith, D.L. (1996) Antiproton Catalyzed Fusion Propulsion for Interplanetary Missions. Proceedings of 32nd AIAA, ASME, SAE, and ASEE, Joint Propulsion Conference and Exhibit, AIAA1996-3068, Lake Buena Vista, Florida, July.

[25] Frisbee, R.H., Brophy, J.R. and VanLandingham, E.E. (1996) Status of NASA’s Advanced Propulsion Technology program. Proceedings of 32nd AIAA, ASME, SAE, and ASEE, Joint Propulsion Conference and Exhibit, 1996-2784, Lake Buena Vista, Florida, July. http://dx.doi.org/10.2514/6.1996-2784

[26] Winterberg, F. (2012) Matter-Antimatter Gigaelectron Volt Gamma Ray Laser Rocket Propulsion. Acta Astronautica, 81, 34-39. http://dx.doi.org/10.1016/j.actaastro.2012.07.001

[27] Ketsdever, A.D., Young, M.P. and Mossman, J.B. (2008) An Overview of Advanced Concepts for Space Access. Proceedings of 44th AIAA, ASME, SAE, and ASEE, Joint Propulsion Conference and Exhibit, AIAA-2008-5121, Hartford, Connecticut, July.

[28] Nakashima, K. and Takabe, H. (2002) Numerical Study of Pair Creation by Ultraintense Lasers. Physics of Plasmas, 9, 1505-1512. http://dx.doi.org/10.1063/1.1464145

[29] Anderson, J.D. (1989) Introduction to Flight. McGraw-Hill, New York.

[30] Sutton, G.P. (1992) Rocket Propulsion Elements. Wiley-Interscience, New York.

[31] Serway, R.A. (1990) Physics for Scientists and Engineers. Saunders College, Philadelphia, Pennsylvania.

[32] http://aries.ucsd.edu/LIB/PROPS/PANOS/w.html

[33] Turns, S.R. (1996) An Introduction to Combustion: Concepts and Applications. McGraw-Hill, New York.

[34] Andrews, D.G. (2004) Interstellar Propulsion Opportunities Using Near-Term Technologies. ActaAstronautica, 55, 443-451. http://dx.doi.org/10.1016/j.actaastro.2004.05.038

[35] http://www.protons.com/protons/who-we-are/about-proton-therapy/index.page 